\title{
ON A PROBLEM OF ERDÖS
}

\section{MING-CHIT LIU}

Erdös posed the following problem in [2, pp. 52-53]: Let $\left\{z_{v}\right\}$ be a sequence with $\left|z_{\nu}\right|=1$ and put

$$
B_{k}=\limsup _{m \rightarrow \infty}\left|\sum_{\nu=1}^{m} z_{\nu}^{k}\right| .
$$

Is it true that $\lim \sup _{k \rightarrow \infty} B_{k}=\infty$ ?

In a subsequent paper [3] Erdös showed that there is an absolute constant $C$ such that we always have $B_{k} / \log k>C$ infinitely often. He also conjected that $B_{k} / k>C$ for infinitely many values of $k$. Recently, Clunie [1] proved that

$$
\limsup _{k \rightarrow \infty} B_{k} / k^{1 / 2}>0,
$$

and there is a sequence $\left\{z_{p}\right\}$ such that $B_{k} \leqq k(k=1,2, \cdots)$.

In this paper we shall prove the following theorem.

THEOREM. Let $\left\{z_{\nu}\right\}$ be any sequence with $\left|z_{\nu}\right|=1$. If $\left\{z_{\nu}\right\}$ contains only a finite number of distinct elements, then for any $\delta>0$,

$$
\limsup _{x \rightarrow \infty} B_{k} / k^{1-\delta}>\frac{1}{5} \text {. }
$$

Let $z_{\nu}=\exp \left[i \phi_{\nu}\right]\left(0 \leqq \phi_{\nu}<2 \pi\right)$. It is easy to see that for any sequence $\left\{z_{p}\right\}$ containing finite distinct elements there exists some $\epsilon>0$ which depends on the sequence $\left\{z_{v}\right\}$ only, such that either

$$
\phi_{\nu}-\phi_{\nu^{\prime}}=0 \text { or }\left|\phi_{\nu}-\phi_{\nu^{\prime}}\right| \geqq \epsilon>0
$$

for all positive integers $\nu, \nu^{\prime}$. So in what follows we shall use $\epsilon$ to denote the maximum positive constant satisfying the above condition.

In order to prove our Theorem we need two lemmas.

LEMMA 1. Let $\left\{\zeta_{\nu}\right\}$ be any sequence containing only a finite number of distinct elements with $\left|\zeta_{\nu}\right|=1(\nu=1,2, \cdots)$. Let $n, k$ be any positive integers and put

Then

$$
S_{k}=\sum_{\nu=1}^{n} \xi_{\nu}^{k}
$$

Received by the editors August 12, 1968. 


$$
\frac{n r^{2}}{\left(1-r^{2}\right)^{2}}-\frac{n^{2}}{\left(1-\cos ^{2} \epsilon\right)^{2}} \leqq \sum_{k=1}^{\infty}\left|S_{k}\right|^{2} k r^{2 k} \quad(0<r<1) .
$$

Proof. Let $z=r e^{i \theta}(0<r<1)$,

$$
P_{n}(z)=\sum_{\nu=1}^{n} \sum_{k=1}^{\infty} k^{1 / 2}\left(\zeta_{\nu} z\right)^{k}=\sum_{k=1}^{\infty} k^{1 / 2} S_{k} z^{k}
$$

We see that

$$
\begin{aligned}
\int_{0}^{2 \pi}\left|P_{n}(z)\right|^{2} d \theta & =\int_{0}^{2 \pi} \sum_{\nu, \nu^{\prime}=1}^{n} \sum_{k, k^{\prime}=1}^{\infty}\left(k k^{\prime}\right)^{1 / 2} \zeta_{\nu}^{k} \bar{\zeta}_{\nu^{\prime}}^{k^{\prime} r^{k+k^{\prime}}} e^{i \theta\left(k-k^{\prime}\right)} d \theta \\
& =2 \pi \sum_{\nu, \nu^{\prime}=1}^{n} \sum_{k=1}^{\infty} k\left(\zeta_{\nu} \bar{\zeta}_{\nu^{\prime}} r^{2}\right)^{k} \\
& =2 \pi \sum_{\nu, \nu^{\prime}=1}^{n} \frac{\zeta_{\nu} \bar{\zeta}_{\nu^{\prime}} r^{2}}{\left(1-\zeta_{\nu} \bar{\zeta}_{\nu^{\prime}} r^{2}\right)^{2}} \\
& =\frac{2 \pi n r^{2}}{\left(1-r^{2}\right)^{2}}+2 \pi \sum_{\nu \neq \nu^{\prime} ; 1 \leq \nu, \nu^{\prime} \leq n} \frac{\zeta_{\nu} \bar{\zeta}_{\nu^{\prime}}, r^{2}}{\left(1-\zeta_{\nu} \bar{\zeta}_{\nu^{\prime}} r^{2}\right)^{2}}
\end{aligned}
$$

and for $\phi_{\nu} \neq \phi_{\nu^{\prime}}$

$$
\begin{aligned}
\left|\frac{\zeta_{\nu} \bar{\zeta}_{\nu}, r^{2}}{\left(1-\zeta_{\nu} \bar{\zeta}_{\nu}, r^{2}\right)^{2}}\right| & \leqq \frac{1}{\inf _{0<r<1}\left|1-\zeta_{\nu} \bar{\zeta}_{\nu} r^{2}\right|^{2}} \\
& =\frac{1}{\left(1-\cos ^{2} \epsilon\right)^{2}}
\end{aligned}
$$

On the other hand, we have

$$
\int_{0}^{2 \pi}\left|P_{n}(z)\right|^{2} d \theta=2 \pi \sum_{k=1}^{\infty}\left|S_{k}\right|^{2} k r^{2 k}
$$

It follows from (1), (2), (3) that

$$
\sum_{k=1}^{\infty}\left|S_{k}\right|^{2} k r^{2 k} \geqq \frac{n r^{2}}{\left(1-r^{2}\right)^{2}}-\frac{n^{2}}{\left(1-\cos ^{2} \epsilon\right)^{2}} \quad(0<r<1) .
$$

The proof of Lemma 1 is completed.

LEMMA 2. Let $\left\{z_{\nu}\right\}$ be any sequence of complex numbers. If $\lim \sup _{k \rightarrow \infty} B_{k} / k^{1-\delta}<C$, for some absolute constants $\delta, C>0$, then there is an integer $k_{1}>0$ which depends on $\left\{z_{p}\right\}, C$ and $\delta$ only, such that given integers $n$ and $\mu(n)$, with $\mu(n) \geqq k_{1}$, we can find $n$ members of $\left\{z_{\nu}\right\}: \zeta_{1}$, $\zeta_{2}, \cdots, \zeta_{n}$ satisfying 


$$
\left|S_{k}\right|=\left|\sum_{\nu=1}^{n} \zeta_{\nu}^{k}\right|<2 C k^{1-\delta} \quad\left(k_{1} \leqq k \leqq \mu(n)\right) .
$$

Proof. From the given hypothesis there is an integer $k_{1}>0$ such that

$$
B_{k}<C k^{1-\delta} \quad\left(k_{1} \leqq k\right) .
$$

Since $B_{k}=\lim \sup _{m \rightarrow \infty}\left|\sum_{\nu=1}^{m} z_{\nu}^{k}\right|$, we see that for each $k \geqq k_{1}$, there exists an integer $N(k)>0$ such that

$$
\left|\sum_{p=1}^{m} \begin{array}{r}
k \\
z_{\nu}
\end{array}\right|<C k^{1-\delta} \quad(m>N(k)) .
$$

For given $n$ choose an integer $N>0$ so that

$$
N \geqq \max _{k_{1} \leqq k \leqq \mu(n)}\{N(k)\} \text {. }
$$

Putting $\zeta_{\nu}=z_{N+\nu}(\nu=1,2, \cdots, n)$, we have

$$
\begin{aligned}
\left|S_{k}\right| & =\left|\zeta_{1}^{k}+\zeta_{2}^{k}+\cdots+\zeta_{n}^{k}\right| \\
& =\left|z_{N+1}^{k}+z_{N+2}^{k}+\cdots+z_{N+n}^{k}\right| \\
& =\left|\sum_{\nu=1}^{N+n} z_{\nu}^{k}-\sum_{\nu=1}^{N} z_{\nu}^{k}\right| \\
& \leqq\left|\sum_{\nu=1}^{N+n} z_{\nu}^{k}\right|+\left|\sum_{\nu=1}^{N} z_{\nu}^{k}\right| \\
& <2 C k^{1-\delta} \quad\left(k_{1} \leqq k \leqq \mu(n)\right) .
\end{aligned}
$$

This completes the proof of Lemma 2 .

We come now to prove our Theorem. For the given sequence $\left\{z_{\nu}\right\}$, suppose lim $\sup _{k \rightarrow \infty} B_{k} / k^{1-\delta}<C$. By virtue of Lemma 2 , there exists a positive integer $k_{1}$ such that for any given $n$ and $\mu(n)$, with $\mu(n) \geqq k_{1}$, there are $\zeta_{1}, \zeta_{2}, \cdots, \zeta_{n} \in\left\{z_{\nu}\right\}$ with

$$
\left|S_{k}\right|<2 C k^{1-\delta} \quad\left(k_{1} \leqq k \leqq \mu(n)\right) .
$$

From Lemma 1 we have

$$
\frac{-n^{2}}{\left(1-\cos ^{2} \epsilon\right)^{2}}+\frac{n r^{2}}{\left(1-r^{2}\right)^{2}} \leqq \sum_{k=1}^{\infty}\left|S_{k}\right|^{2} k r^{2 k}
$$

$$
\begin{aligned}
& =\left(\sum_{k=1}^{k_{1}}+\sum_{k>k_{1}}^{\mu(n)}+\sum_{k>\mu(n)}^{\infty}\right)\left|S_{k}\right|^{2} k r^{2 k} \\
& =T_{1}+T_{2}+T_{3}, \text { say. }
\end{aligned}
$$


Since $\left|S_{k}\right| \leqq n$, we have

$$
\begin{aligned}
T_{1}=\sum_{k=1}^{k_{1}}\left|S_{k}\right|^{2} k r^{2 k} & \leqq n^{2} \sum_{k=1}^{k_{1}} k<n^{2} k_{1}^{2}, \\
T_{3}=\sum_{k>\mu(n)}^{\infty}\left|S_{k}\right|^{2} k r^{2 k} & \leqq n^{2} \sum_{k>\mu(n)}^{\infty} k r^{2 k} \\
& \leqq n^{2} \mu(n) \frac{r^{2 \mu(n)}}{\left(1-r^{2}\right)^{2}} .
\end{aligned}
$$

Let $A_{k}^{\alpha}$ be the Cesàro numbers of order $\alpha(0<\alpha)$, we have

$$
\begin{aligned}
\sum_{k=0}^{\infty} A_{k}^{\alpha}{ }^{2 k} & =\frac{1}{\left(1-r^{2}\right)^{1+\alpha}} \quad(0<r<1), \\
A_{k}^{\alpha} & =\frac{k^{\alpha}}{\Gamma(1+\alpha)}\{1+o(1)\} \quad \text { as } k \rightarrow \infty .
\end{aligned}
$$

See [4, Vol. I, pp. 76-77]. Assume that $k_{1}$ in (4) is large such that for every $k \geqq k_{1}$ we have $A_{\mathbf{z}}^{3-\delta} \Gamma(4) \geqq k^{3-\delta}$. From (4) it follows that

$$
\begin{aligned}
T_{2} & =\sum_{k>k_{1}}^{\mu(n)}\left|S_{k}\right|^{2} k r^{2 k} \leqq 4 C^{2} \sum_{k>k_{1}}^{\mu(n)} k^{2-\delta} r^{2 k} \\
& \leqq 4 C^{2} \Gamma(4) \sum_{k=0}^{\infty} A_{k}^{3-\delta} r^{2 k}=24 C^{2} \frac{1}{\left(1-r^{2}\right)^{4-\delta}} .
\end{aligned}
$$

From (5), (6), (7) we have

(8) $\frac{-n^{2}}{\left(1-\cos ^{2} \epsilon\right)^{2}}+\frac{n r^{2}}{\left(1-r^{2}\right)^{2}} \leqq n^{2} k_{1}^{2}+\frac{24 C^{2}}{\left(1-r^{2}\right)^{4-\delta}}+\frac{n^{2} \mu(n) r^{2 \mu(n)}}{\left(1-r^{2}\right)^{2}}$.

Put $1 /\left(1-r^{2}\right)=n^{1 /(2-\delta)}$ and $\mu(n)=n^{2}$. Then we have

$$
\frac{n}{\left(1-r^{2}\right)^{2}}=\frac{1}{\left(1-r^{2}\right)^{4-\delta}}=n^{2+(8 /(2-\delta))}
$$

and

$$
\frac{n^{2} \mu(n) r^{2 \mu(n)}}{\left(1-r^{2}\right)^{2}}<n^{6}\left(1-n^{-1 /(2-\delta)}\right)^{n^{2}}<n^{6} e^{-n}=o(1)
$$

as $n \rightarrow \infty$. Thus, taking $n \rightarrow \infty$, from (8) we have

$$
1 \leqq 24 C^{2} \text {. }
$$

This implies that 


$$
\underset{k \rightarrow \infty}{\lim \sup } B_{k} / k^{1-\delta} \geqq 1 / \sqrt{ } 24>1 / 5 \quad(\delta>0) .
$$

The proof is completed.

My thanks are due to the referee for some useful remarks and improvements in this paper.

\section{REFERENCES}

1. J. Clunie, On a problem of Erdös, J. London Math. Soc. 42 (1967), 133-136.

2. P. Erdös, Problems and results on Diophantine approximations, Compositio Math. 16 (1964), 52-65.

3. - Some remarks on number theory, Israel J. Math. 3 (1965), 6-12.

4. A. Zygmund, Trigonometric series, Cambridge Univ. Press, New York, 1959.

UNIVERSITY OF HONG KONG 